\title{
Efficient Diabetic Retinopathy Space Subjective Fuzzy Clustering In Digital Fundus Images
}

\author{
Padmalal. S, C.Nelson Kennedy Babu \\ Principal, CMS Polytechnic College, Namakkal 637003 \\ Principal, CMS College of Engineering, Namakkal 637003
}

\begin{abstract}
Diabetic Retinopathy $(D R)$ is a very rigorous and extensive eye disease which causes blindness in diabetic patients. DR primary cause for blindness is not for the reason that it has the highest occurrence but it often remains unobserved until severe vision loss occurs. Diabetic Retinopathy is characterized by changes in the retina that comprise of change in blood vessel diameter, micro aneurysms, lipid, and protein deposits with cotton coat spot. It depends on the appearance, hemorrhages and new vessel growth. For the further improvement in detection of DR, a new technique named Space Subjective Fuzzy Clustering Method (SSFC) is introduced which used for the extraction of retinal vessels. Initially the segmentation of digital fundus images takes place and then SSFC method introduced with mathematical study of shape. In mathematical study of shape, the digital fundus image is smoothed and strengthened so that the retinal vessels are enhanced and the backdrop information is suppressed. The Space Subjective Fuzzy Clustering algorithm is then employed to the enhanced segmented image. After the SSFC, a refinement process is used to decrease the fragile edges noise, and the final results of the retinal vessels of DR are accordingly achieved. The performance of the SSFC method is compared with some existing segmentation methods using Gold Standard Database, STARE, and DRIVE Dataset. Performance of Space Subjective Fuzzy Clustering Method is measured in term of segmentation accuracy ratio and clustering efficiency. The approach has been tested on a series of digital fundus images, and experimental results show that our technique is hopeful and effective.
\end{abstract}

Keywords: Diabetic Retinopathy, Space Subjective Fuzzy Clustering, Retinal Vessels, Digital Fundus Images, Clustering Efficiency, Purification, Segmentation Accuracy Ratio.

\section{Introduction}

Retinal images of humans play a significant role in the detection and diagnosis of many eye diseases. Some diseases such as glaucoma diabetic retinopathy and macular degeneration are very serious for which they can lead to blindness if they are not detected in time and correctly. Therefore, automatic detection for retinal images is necessary and among them the detection of retinal vessels is most important. The information about retinal vessels such as length, width, and branching pattern can not only supply information on pathological changes but can also help to grade diseases rigorousness to automatically diagnose the diseases. However, physical detection of retinal vessels is much more complex since the retinal vessels image are compound and with little contrast.

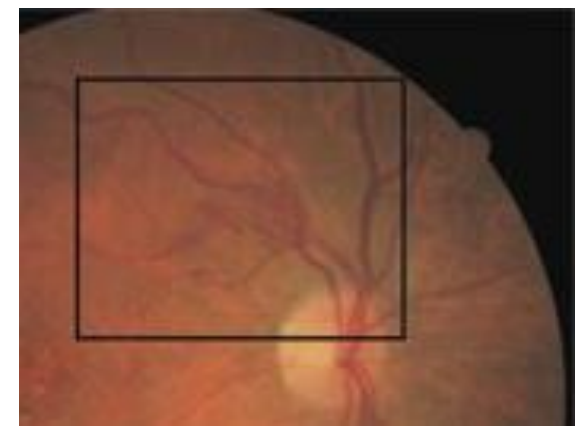

Fig 1.1 Retinal vessels Image with macular hole

The above Fig 1.1 illustrates the image with macular hole. In addition, there are usually a number of retinal images to judge a disease. Hence, a physical measurement becomes annoying which is a reliable and automatic method for extracting and measuring the retinal images vessels. In the past years, many approaches for extracting retinal image vessels have been applied but not in an extensive way. The previous method typically uses a two-dimensional linear structural constituent with Gaussian cross-profile section as rotated into three dimensions to identify the cross profile of the retinal vessels. The resulted image is at last threshold to produce a binary segmentation. 
Another technique for vessel extraction is the vessel tracking method in which each vessel segment is defined by three attributes namely direction, breadth, and middle point. The thickness distribution of the cross section of a blood vessel is predictable using a Gaussian shaped function. Each segment are identified using a explore system which keeps follow of the middle of the vessel and makes some decisions about the future path of the vessel based on certain vessel properties. However, the vessel-tracking method requires user interference and perplexed by vessel crossing and bifurcations. Balint Antal., and Andras Hajdu., 2010 propose an ensemblebased framework to improve micro aneurysm detection. Unlike the well-known approach of considering the output of multiple classifiers, a combination of internal components of micro aneurysm detectors, namely preprocessing methods and candidate extractors used and plan to further improve the performance.

Akara Sopharak.,et.Al, 2011 investigates a position of optimally adjusted morphological operators used for micro aneurysm detection on non-dilated pupil and low-contrast retinal images. The detected micro aneurysms are validated by comparing with ophthalmologists' hand-drawn ground-truth. A negative aspect of these methods is that the large-scale of retina vessels cannot be applied to the classification until the efficient segmentation is performed. To overcome the drawback of Diabetic Retinopathy detection, presented a novel approach for retinal vessel extraction using mathematical study of shape and a Space Subjective Fuzzy Clustering method.

The SSFC method involves two main steps. In the first step, gray mathematical study of shape theories are given to smooth and strengthen the retinal images in order to remove the background and improve the brightness of retinal vessels using segmentation. In the subsequent step, a Space Subjective Fuzzy Clustering method is employed to remove retinal vessels followed by a refinement procedure. The final results of the retinal vessels are then achieved using efficient clustering.

We provide here an overview of Space Subjective Fuzzy Clustering Method. The rest of this paper is arranged as follows: Section 2 describes about the different surveys. Section 3 introduces architecture diagram of the proposed scheme. Section 3.1 and 3.2 describes about proposed method; Section 4 shows the evolution and experimental evaluation; Section 5 evaluated the results and discuss about it. Section 6 describes conclusion and outlook.

\section{Literature Review}

Diabetic Retinopathy is a medical condition where the retina is scratched because fluid leaks from blood vessels into the retina. The presence of hemorrhages in the retina is the initial indication of diabetic retinopathy. Parisut Jitpakdee., et.Al., 2012 review the techniques, algorithms, and methodologies used for the discovery of hemorrhage from diabetic retinopathy retinal images. Anderson Rocha.,et.Al., 2012 present an algorithm to notice the presence of diabetic retinopathy (DR) correlated lesions from fundus images based on a universal logical approach that is competent of identifying both red and bright lesions devoid of requiring specific pre- or post-processing.

Various aspects and stages of retinopathy are analyzed by investigative the colored retinal images. Micro aneurysms are small secular pouches caused by local swelling of capillary walls and come into view as small red dots. Neera Singh., and Ramesh Chandra Tripathi., 2010 proposed walls are slim and break simply to cause hemorrhages. Hard exudates are yellow lipid deposits which come out as bright yellow lesions. The bright circular district from where the blood vessels originate is called the optic disk. The fovea defines the center of the retina, and is the region of highest visual acuity.

N. Venkata Krishna.,et.AL., 2013 propose a mixture of interior components of micro aneurysm detectors, specifically preprocessing methods and applicant extractors. Since micro aneurysm detection is important in diabetic retinopathy grading. Keerthi Ram., et.Al., 2010 present a new approach for routine MA detection from digital color fundus images. We devise MA detection as a problem of objective detection from clutter, where the likelihood of happening of target is noticeably lesser compared to the clutter. A consecutive rejection-based policy is planned to gradually inferior the numeral of clutter responses.

Nathan Silberman.,et.Al., 2010 expand an automatic system to notice diabetic retinopathy from retinal images. The automated diabetic retinopathy problem is a firm computer vision problem whose goal is to notice features of retinopathy, such as hemorrhages and exudates, in retinal color fundus images. Simon Barriga.,et.Al., 2011 introduces the three stages of the steps namely, retinal images of the patient are captured with a non mydriatic fundus camera. Next, the images are analyzed with our automatic DR screening software. Final step involves the medical evaluation process.

Honggang Yu.,et.Al., 2012 presents a scheme that can mechanically decide whether the excellence of a retinal image is enough for computer-based diabetic retinopathy (DR) screening. The system integrates global histogram features, textural features, and vessel density, as well as a local non-reference perceptual sharpness metric. Lingfeng Yu., and Zhongping Chen., 2010 show the use of Doppler variance standard deviation imaging for 3-D in vivo angiography in the human eye. In addition to the usual optical Doppler tomography velocity and structural images, use the variance of blood flow speed to chart the retina and choroid vessels. Variance imaging 
is focus to bulk motion artifacts as in phase-resolved Doppler imaging, and a histogram-based method is planned for bulk-motion correction in variance imaging.

Jaspreet Kaur., and Dr.H.P.Sinha., 2012 develops an automated screening systems for diabetic retinopathy. Optic disc boundary and localization of macula are the two features of retina were essential for the detection of exudates and also significant the harshness of the diabetic maculopathy. According to the prior information, the diameter of optic disc in a normal retinal image, connected components and iterative thresholding was used to position optic disc.

Ragav Venkatesan.,et.Al., 2012 propose a modified color auto correlogram feature (AutoCC) with low dimensionality that is spectrally tuned towards DR images. Recognizing the fact that the images with or without MA are usually dissimilar only in small, localized regions. It introduces the perception of utilizing a multiclass, multiple-instance learning framework for performing the categorization task by means of the planned attribute.

Adapting to particular aspect of DR recognition with minimal cost developed a technique named Space Subjective Fuzzy Clustering Method in digital fundus image.

\section{Space Subjective Fuzzy Clustering Technique In Digital Fundus Images}

Digital Fundus images usually have pathological noise and various texture backgrounds, which may cause difficulties in extraction. Conversely, the line type feature of the retina vessels is not changed when the backdrop textures of the image are different. Based on SSFC technique, the outline of our enhancing method is described in Fig 3.1

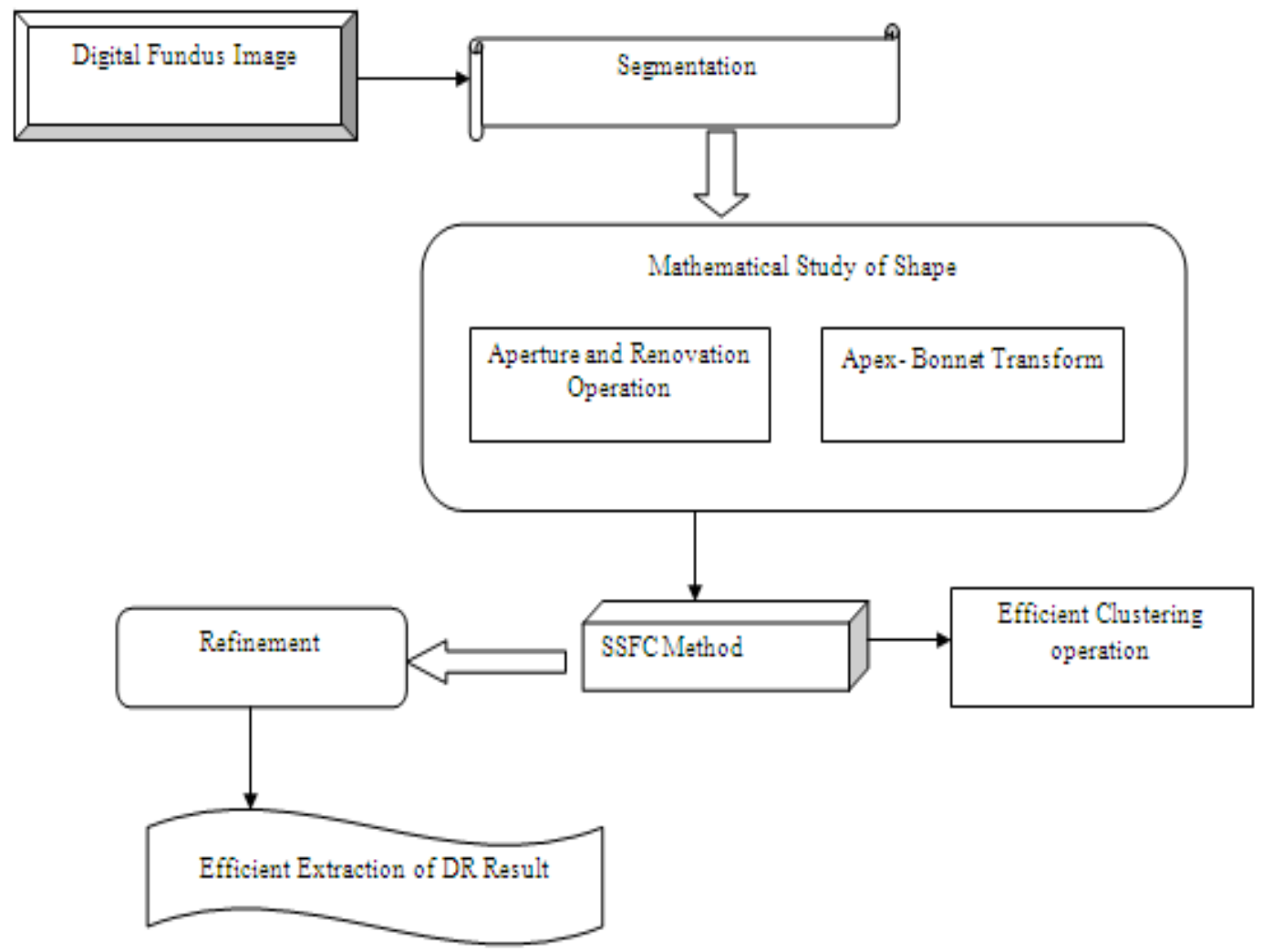

Fig 3.1 Architecture Diagram of Space Subjective Fuzzy Clustering Method

The above Fig 3.1 takes the digital fundus images which interior surface of the eye, including the retina, optic disc, macula, and posterior pole. Digital Fundus Image is used by optometrists, ophthalmologists, and skilled medical professionals for monitoring succession of a disease, diagnosis of a disease in screening programs. Segmentation process is carried out in the digital fundus images to highly contrast the disease. Firstly, in order to remove the noise, a gray aperture operation and an aperture by renovation with a linear structuring element are employed to the original image at various orientations.

Secondly, the Apex-Bonnet transform combined with renovation opening and ultimate operations are proposed to make stronger the smoothed image by iteratively filtering the digital fundus image. The Space Subjective Fuzzy Clustering Method is employed to remove retinal vessels followed by a refinement procedure. The final results of the retinal vessels are then achieved using efficient clustering and extraction of DR result. 


\subsection{Retinal Image Smoothing and Removing Noise}

SSFC Gray mathematical study of shape theory is a kind of non-linear theory based on set theory and is advantageous to geometrically describe an image. The theory can be used to extract gray linear properties by convolving the SSFC image with a line type-structuring element at various orientations. The key in the gray mathematical study of shape process is to select the structuring element. It uses linear structuring elements according to the procession type property of retina vessels. However, it is important to note that an SSFC aperture operation used by a linear structuring element will remove a retina vessel or some parts of it when the retina vessels in the image. On the contrary, when the structuring element of SSFC method and the retina vessel have equivalent directions, the retina vessel will never be changed.

Therefore, SSFC technique convolves the digital fundus image with the linear structuring element at various orientations to get the maximum response. To gratify the need of extracting larger retina vessels to identify the diabetic retinopathy at the earlier stage using the digital fundus images, the length of structuring elements is selected to be close to the diameter value of the largest vessels. In experimental set up, in order to save the time spent on the segmentation process of SSFC technique, the size of the digital fundus image is now reduced to $220 \times 256 \times 8$. The diameter of the major retina vessels is approximately measured in pixels. Hence, each structuring element contains both the length and breadth of the pixel. The calculation process of SSFC is defined as follows:

$$
\begin{gathered}
\gamma_{M=\delta_{M}}\left(\in_{M}(L)\right. \\
D=\max _{i=1,2 \mathrm{~m} 12}\left\{\gamma_{M i}(D o)\right\} \\
D o p=\gamma_{D 0}^{r \theta c}(D)=\sup _{S \in N}\left(\Delta_{D o}^{s}\right) \\
\epsilon_{M} \min _{L+M(L)}(D o(L)) \ldots \\
\gamma_{M} \max _{L+M(L)}(D o(L)) \ldots \ldots
\end{gathered}
$$

Structuring elements of SSFC are applied here to perform an aperture operation $\gamma \mathrm{Mi}$ on the original image $\mathrm{D}_{0}$. This aperture operation consists of two steps. The first step is to corrode the digital fundus image defined as $\varepsilon \mathrm{M}$, and the second step is to widen the digital fundus image defined as $\delta \mathrm{M}$. The maximum response of all directions is defined as the aperture digital fundus image D. The reconstructed image $\mathrm{D}$ op that is the smoothed image obtained by carrying out an opening by reconstruction $\gamma_{D 0}^{r e c}$ to the original image D0 and the aperture image S. It has a few retina vessel tumors in the vessels. Before smoothing and removing noise, the color of the original image is reversed. Eqn. (3) and (5) are applied to the reversed image to get the smoothen image.

\subsection{Retina Vessels strengthen by removing Backdrop}

After the image has been smoothed, the Apex -Bonnet transform is applied to strengthen the retina vessels in the digital fundus image by choosing appropriate structuring elements. Here, the Apex -Bonnet transform of SSFC technique is applied to the smoothed image at all directions, and the computational results of the directions are summed up to increase the gray difference between the retina vessels and the backdrop. Usually, retina vessels in digital fundus image might be segmented with an effortless verge on $\mathrm{D}_{\text {sum }}$.

$$
\mathrm{D}_{\text {sum }}=\in_{i=1}^{12}\left(\text { Dop }-\gamma_{M i}(D o)\right)
$$

However, most images contain noisy data requiring a further treatment such as SSFC technique to completely remove the noise and smoothes the digital fundus image. Then the operations of aperture by renovation and ultimate by renovation are applied to the digital fundus image to enhance the image iteratively.

\subsection{SSFC method for retina vessel extraction}

When the retinal vessels have been enhanced in SSFC method, the next step is to extract the retina vessels from the digital fundus image. However, it is essential that there is some ambiguity in influential the exact position of a retina vessel segment in the image due to the image-generation process. The Space Subjective Fuzzy Clustering is introduced has a powerful tool to tackle the difficulty in previous image segmentation process. 


$$
I_{S S F C}=\in_{j=1}^{c} \in_{i=1}^{m}\left(u_{i, j}\right) w s^{2}\left(x_{j_{2}} v_{i}\right)
$$

Where, $X=\left\{x_{1}, x_{2}, \ldots, x_{m}\right\} \subseteq S p$ is the data set in the $\mathrm{p}$ dimensional vector space,

$\mathrm{m}$ - Number of data items,

c - Number of clusters with $2 \leq \mathrm{c}<\mathrm{m}$,

$u_{i, j}$ - Degree of space subjective fuzzy membership of $x_{j}$ to the $i^{\text {th }}$ cluster,

$\mathrm{w}$ - Weighting exponent on each space subjective fuzzy membership,

$v_{i}$-Prototype of the centre cluster $i$,

$\mathrm{s}^{2}\left(\mathrm{x}_{\mathrm{j}}, \mathrm{v}_{\mathrm{i}}\right)$ - Distance measure between object $\mathrm{x}_{\mathrm{j}}$ and cluster centre $\mathrm{v}_{\mathrm{i}}$.

A minimum of the Space Subjective Fuzzy Clustering (SSFC) function are obtained with the iterative process.

\subsection{Algorithm for SSFC technique:}

Generic Steps of the detection of diabetic retinopathy at the earlier stage using the Space Subjective Fuzzy Clustering in digital fundus image.

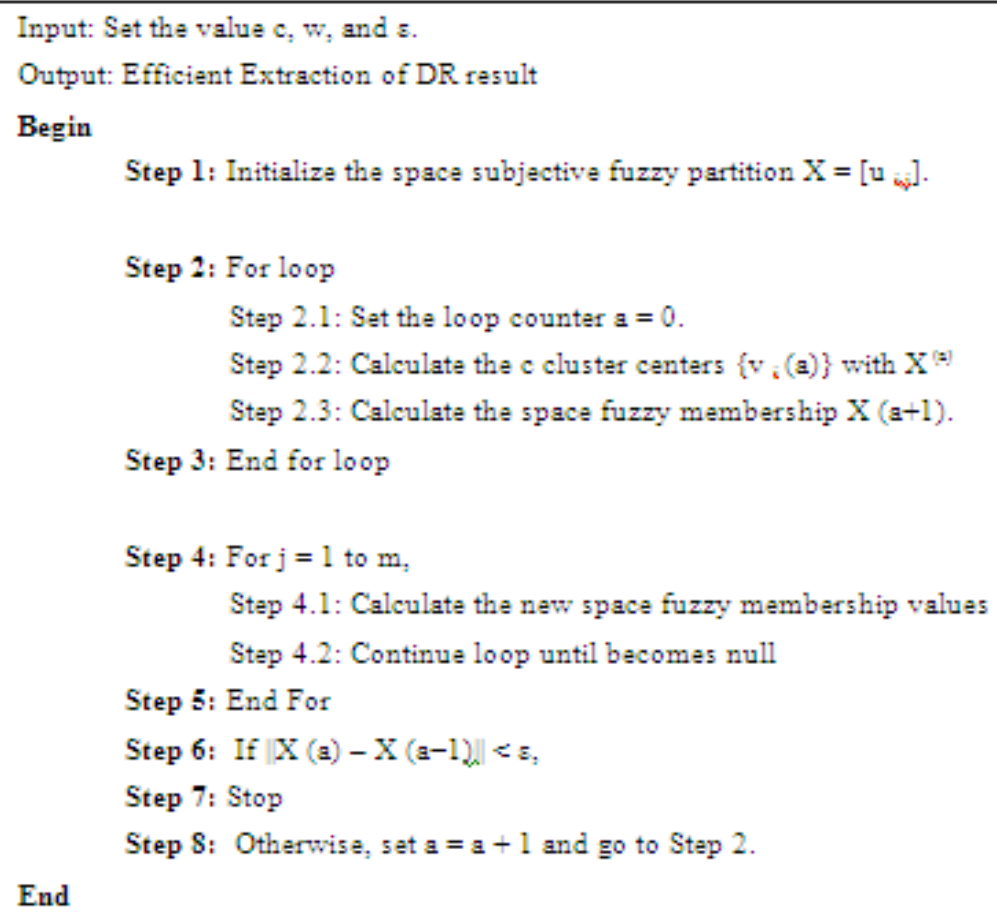

The above algorithm has a crisp description of segmentation process and the space subjective clustering process using the digital fundus images. The fuzzy partition is initialized in the ' $\mathrm{X}$ ' in which the maximum membership procedure is most important. The procedure assigns object $\mathrm{j}$ to class $\mathrm{C}$ with the highest space subjective fuzzy membership. With this procedure, the fuzzy digital fundus image is then converted to a crisp one in SSFC method. However, since there are some weak edges and noise in the digital fundus image after this fuzzy extraction, used a refinement procedure to purify the result and the final extraction is achieved in this case of SSFC method.

\section{Experimental Evaluation}

Diabetic Retinopathy space subjective Fuzzy Clustering in Digital Fundus Image is implemented in MATLAB. The Digital Fundus images are taken from the Gold Standard Database for Evaluation of Fundus Image Segmentation Algorithms, DRIVE Dataset and STARE (STructured Analysis of the Retina) Project database.

Gold Standard database has been recognized by a joint research group to hold up relative studies on automatic segmentation algorithms on retinal fundus images. The database will be iteratively comprehensive and the webpage will be improved. It would like to help researchers in the assessment of segmentation algorithms and encourage anyone working with segmentation algorithms who establish our database useful to 
send us their evaluation results. This way can extend our database of algorithms with the given results to keep it always up-to-date.

The Digital Retinal Images for Vessel Extraction (DRIVE) database has been well-known to allow comparative studies on segmentation of blood vessels in retinal images. The research community is invited to test their algorithms on this database and share the results with other researchers through this web site. The instruction established on downloading the database and uploading results and the results of various methods can be inspected. The photographs for the DRIVE database were obtained from a diabetic retinopathy screening program. The screening population consisted of 400 diabetic subjects between 25-90 years of age. Forty photographs have been arbitrarily selected, 33 do not show any sign of diabetic retinopathy and 7 show signs of mild untimely diabetic retinopathy. Each image has been JPEG compressed.

The STARE (STructured Analysis of the Retina) Project contains at the moment 15 images of healthy patients, 15 images of patients with diabetic retinopathy and 15 images of glaucomatous patients. Binary gold standard vessel segmentation images are available for each image. The masks determining field of view (FOV) are provided for particular datasets. The gold standard data is generated by a collection of experts working in the field of retinal image analysis and clinicians from the cooperated ophthalmology clinics. The performance of the proposed Diabetic Retinopathy Space Subjective Fuzzy Clustering in Digital Fundus Image is measured in terms of

- Segmentation Accuracy ratio

- Average Sensitivity Value

- Clustering Efficiency

\section{Results And Discussion}

The performance of detecting the diabetic retinopathy using the Space Subjective Fuzzy Clustering in Digital Fundus Image is evaluated. The below table and graph describes the performance of the Space Subjective Fuzzy Clustering in Digital Fundus Image (SSFC) method with the Ensemble-based System for Micro aneurysm Detection (ESMD) technique.

Segmentation Accuracy ratio: Segmentation Accuracy refers to how far a determined experimental value may be from the actual value. This is in distinct contrast to precision, which refers to how close experimental data is grouped together with subsequent repetitions.

$$
\text { Accuracy }=
$$

TPR+TNR

\section{TPR+TNR+FPR+FNR}

Where, TPR-True Positive Rate

TNR- True Negative Rate

FPR-False Positive Rate

FNR- False Negative Rate

\begin{tabular}{|c|c|c|}
\hline \multirow{2}{*}{ No. of images } & \multicolumn{2}{|c|}{ Segmentation Accuracy Ratio } \\
\cline { 2 - 3 } & Proposed SSFC method & ESMD Technique \\
\hline 5 & 0.98 & 0.66 \\
\hline 10 & 0.97 & 0.68 \\
\hline 15 & 0.95 & 0.69 \\
\hline 20 & 0.95 & 0.69 \\
\hline 25 & 0.94 & 0.70 \\
\hline 30 & 0.92 & 0.72 \\
\hline 35 & 0.93 & 0.73 \\
\hline
\end{tabular}

Table 5.1 No. of images vs. Segmentation Accuracy ratio

The table 5.1 explains the segmentation accuracy ratio based on the number of images in the Gold Standard database. The segmentation accuracy ratio of the Space Subjective Fuzzy Clustering method is compared with the Ensemble-based System for Micro aneurysm Detection (ESMD) technique.

Fig 5.1 describes the segmentation accuracy ratio on the Space Subjective Fuzzy Clustering (SSFC) method by removing the noise and smoothened the image surface using the mathematical study of the shape. The SSFC method on the gold database performs the efficient segmentation process on the up to date information. The segmentation is the initial process in the SSFC method. 


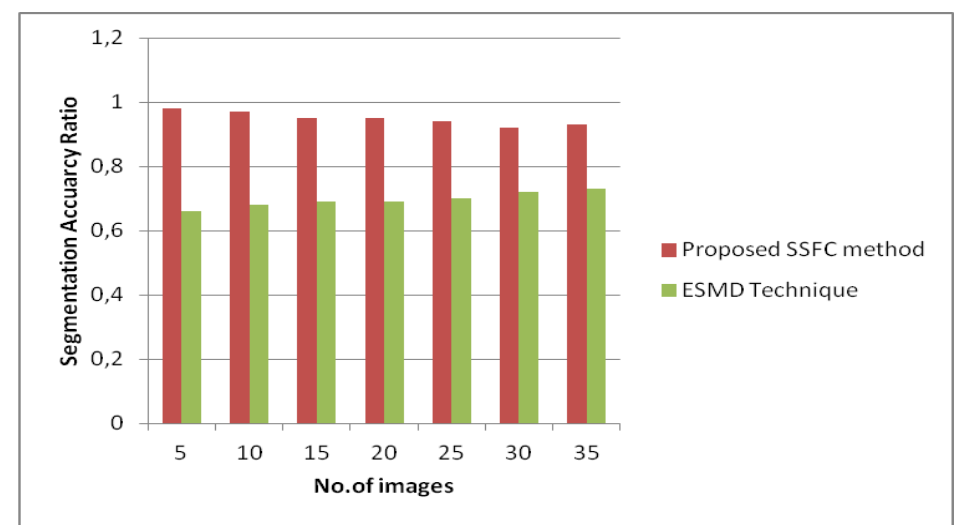

Fig 5.1 No. of images vs. Segmentation Accuracy ratio

The outcome of the SSFC method is approximately $65-75 \%$ higher than present Ensemble-based System for Micro aneurysm Detection (ESMD) technique.

Average Sensitivity Value: Sensitivity measures the amount of actual positives which are correctly identified from the percentage of sick people who are correctly identified as having the disease.

Sensitivity $=\quad \mathrm{TPR}$

$\mathrm{TPR}+\mathrm{FNR}$

\begin{tabular}{|c|c|c|}
\hline \multirow{2}{*}{$\begin{array}{c}\text { False positive per } \\
\text { Image }\end{array}$} & \multicolumn{2}{|c|}{ Average Sensitivity Value } \\
\cline { 2 - 3 } & Proposed SSFC method & ESMD Technique \\
\hline 10 & 1.3 & 0.5 \\
\hline 20 & 2.2 & 0.9 \\
\hline 30 & 3.0 & 1.2 \\
\hline 40 & 3.5 & 1.5 \\
\hline 50 & 4.4 & 1.9 \\
\hline 60 & 4.9 & 2.2 \\
\hline 70 & 5.5 & 2.8 \\
\hline 80 & 6.0 & 3.2 \\
\hline
\end{tabular}

Table 5.2 False positive per Image vs. Average Sensitivity Value

The above table 5.2 describes the average sensitivity value based on the false positive per image. The Average Sensitivity Value is measured in the Digital Retinal Images for Vessel Extraction dataset. The Average Sensitivity Value of the Space Subjective Fuzzy Clustering method is compared with the Ensemble-based System for Micro aneurysm Detection (ESMD) technique.

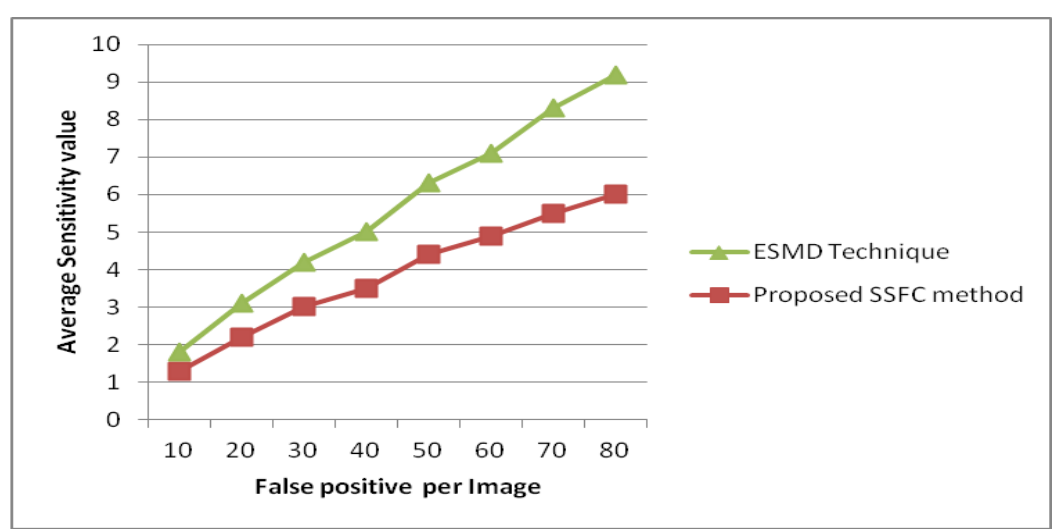

Fig 5.2 False positive per Image vs. Average Sensitivity Value

Fig 5.2 describes the sensitivity value on the false positive per image. The sensitivity refers to the information about the third persons diseases are maintained in the privacy table. The observed performance from the SSFC method from the Digital Retinal Images for Vessel Extraction (DRIVE) dataset reveals the 
highest privacy when compared with the Ensemble-based System for Micro aneurysm Detection (ESMD) technique. The variance in sensitivity value is approximately $45-50 \%$ higher in the Digital Retinopathy Space Subjective Fuzzy Clustering method.

Clustering Efficiency: It is defined as the amount of images efficiently clustered depending on the similar rules. It is measured in terms of percentage (\%).

$$
\text { Clustering Efficiency }=\left[\begin{array}{l}
\text { No. of images clustered } * 100 \\
\text { Total no. of digital fundus images }
\end{array}\right]
$$

\begin{tabular}{|c|c|c|}
\hline \multirow{2}{*}{$\begin{array}{c}\text { No. of digital } \\
\text { fundus images }\end{array}$} & \multicolumn{2}{|c|}{ Clustering Efficiency (\%) } \\
\cline { 2 - 3 } & $\begin{array}{c}\text { Proposed } \\
\text { SSFC } \\
\text { method }\end{array}$ & ESMD Technique \\
\hline 25 & 93 & 66 \\
\hline 50 & 94 & 68 \\
\hline 75 & 95 & 69 \\
\hline 100 & 96 & 70 \\
\hline 125 & 96 & 67 \\
\hline 150 & 96 & 67 \\
\hline 175 & 97 & 69 \\
\hline 200 & 95 & 71 \\
\hline
\end{tabular}

Table 5.3 No. of digital fundus images vs. Clustering Efficiency

Fig 5.3, described the clustering efficiency based on the digital fundus image. The Average Sensitivity Value of the Space Subjective Fuzzy Clustering method performance is measured in the STARE (STructured Analysis of the Retina) Project and the comparison done with the Ensemble-based System for Micro aneurysm Detection (ESMD) technique.

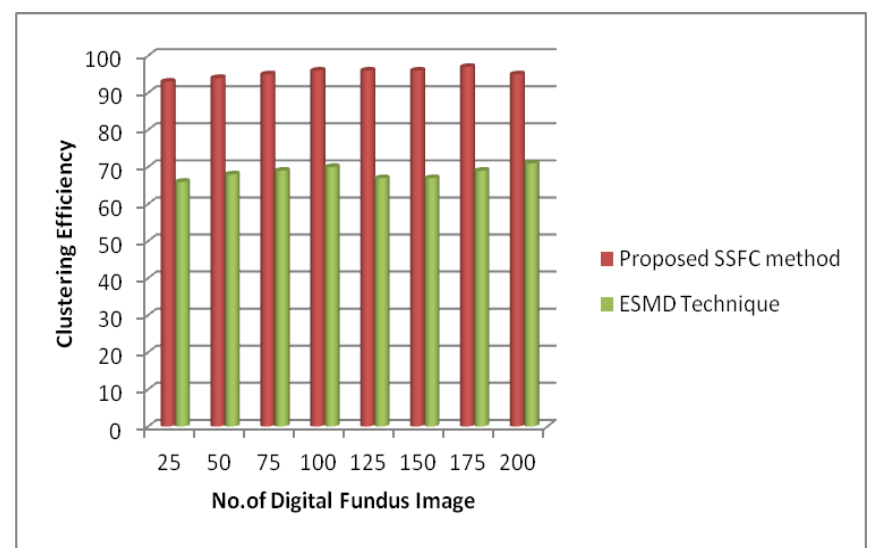

Fig 5.3 No. of digital fundus images vs. Clustering Efficiency

The performance graph of the clustering efficiency is compared with the digital fundus images to show the efficiency in result. It is examined using the STARE (STructured Analysis of the Retina) Project images by producing the excellent result with efficient DR clustering. However, the false positive rates of SSF clustering are less than those of the second specialist, which means that SSFC method can yield smaller retina vessels than the second specialist. The variance in the STARE dataset evaluation would be approximately 15-20\% higher clustering efficiency in Space Subjective Fuzzy Clustering in Digital Fundus Image (SSFC) method when compared with the Ensemble-based System for Micro aneurysm Detection (ESMD) technique.

Finally, it is being observed that the Diabetic Retinopathy space subjective Fuzzy Clustering eradicates the noise occurring in the digital fundus images and smoothes the image surface uses the mathematical study of shape. It is analyzed through the Aperture and Renovation Operation and Apex- Bonnet Transform mathematical study of shape in Gold Standard Database for Evaluation Segmentation, DRIVE Dataset and STARE (STructured Analysis of the Retina) Project database. 


\section{Conclusion}

Space Subjective Fuzzy Clustering Method (SSFC) in this phase is used for the extraction of retinal vessels. The method is developed by using mathematical study of shape combined with the space subjective fuzzy clustering algorithm. Initially, it first employed segmentation to smooth and strengthen the digital fundus images as well as to suppress the backdrop information. Then, the Space Subjective Fuzzy Clustering algorithm is applied to the enhanced retinal images. Finally, refinement procedure is performed to purify the digital fundus image by easily detect the diabetic retinopathy disease. The detection results obtained from the SSFC method are compared with those of the standard segmentation methods using Gold Standard Database, STARE, and DRIVE Dataset. Performance of Space Subjective Fuzzy Clustering Method is measured in term of segmentation accuracy ratio, average sensitivity value and clustering efficiency. Both qualitative and quantitative experiments on digital fundus images indicate that the SSFC approach is effective and can produce identical results and yield a $80-90 \%$ higher accuracy ratio and a lower misclassification ratio than the manual extractions.

\section{References}

[1]. Balint Antal., and Andras Hajdu., "An Ensemble-based System for Microaneurysm Detection and Diabetic Retinopathy Grading," IEEE TRANSACTIONS ON BIOMEDICAL ENGINEERING, 2010

[2]. Akara Sopharak., Bunyarit Uyyanonvara., and Sarah Barman, “Automatic Microaneurysm Detection from Non-dilated Diabetic Retinopathy Retinal Images Using Mathematical Morphology Methods,” IAENG International Journal of Computer Science, 2011

[3]. Parisut Jitpakdee., Pakinee Aimmanee., Bunyarit Uyyanonvara., "A Survey on Hemorrhage Detection in Diabetic Retinopathy Retinal Images," 978-1-4673-2025-2/12/\$31.00, IEEE, 2012

[4]. Anderson Rocha., Tiago Carvalho., Herbert F. Jelinek., Siome Goldenstein., and Jacques Wainer., "Points of Interest and Visual Dictionaries for Automatic Retinal Lesion Detection," IEEE TRANSACTIONS ON BIOMEDICAL ENGINEERING, 2012

[5]. Keerthi Ram., Gopal Datt Joshi., and Jayanthi Sivaswamy., "A Successive Clutter-Rejection based Approach for Early Detection of Diabetic Retinopathy," IEEE TRANSACTIONS ON BIOMEDICAL ENGINEERING, 2010

[6]. Simon Barriga., "A Hybrid Top-Down Approach to Diabetic Retinopathy Screening,", 2011

[7]. Lingfeng Yu., Zhongping Chen., "Doppler variance imaging for three-dimensional retina and choroid angiography," Journal of Biomedical Optics, 2010

[8]. N. Venkata Krishna., N. Venkata Siva Reddy., M. Venkata Ramana., E. Prasanna Kumar., "The Communal System for Early Detection Microaneurysm and Diabetic Retinopathy Grading Through Color Fundus Images," International Journal of Scientific Engineering and Technology (ISSN : 2277-1581) Volume 2, Issue 4, 2013

[9]. Ragav Venkatesan., Parag Chandakkar., Baoxin Li., and Helen K. Li., "Classification of Diabetic Retinopathy Images Using MultiClass Multiple-Instance Learning Based on Color Correlogram Features," 2012

[10]. Neera Singh., Ramesh Chandra Tripathi., "Automated Early Detection of Diabetic Retinopathy Using Image Analysis Techniques," International Journal of Computer Applications (0975 - 8887) Volume 8- No.2, 2010

[11]. Nathan Silberman., Kristy Ahlrich., Rob Fergus., and Lakshminarayanan Subramanian., "Case for Automated Detection of Diabetic Retinopathy," Association for the Advancement of Artificial Intelligence, 2010

[12]. Honggang Yu., Carla Agurto., Simon Barriga., Sheila C. Nemeth., Peter Soliz., Gilberto Zamora., "Automated image quality evaluation of retinal fundus photographs in diabetic retinopathy screening," 978-1-4673-1830-3/12/\$31.00 , IEEE, 2012

[13]. Jaspreet Kaur., Dr.H.P.Sinha., “Automated Localisation of Optic Disc and Macula from Fundus Images," International Journal of Advanced Research in Computer Science and Software Engineering., Volume 2, Issue 4, 2012 